\title{
$X_{\max }$ reconstruction for EAS with energy above $10^{16} \mathrm{eV}$ from responses of tracking Cherenkov detectors
}

Mokhnachevskaya V.P.1, Egorov Yu.A., Knurenko S.P., Sleptsov I.Ye., Petrov Z.E.

$Y$ u. G. Shafer Institute of cosmophysical research and aeronomy SB RAS

E-mail: valyamokheyandex.ru

This work describes the system of tracking detectors operating at the Yakutsk complex EAS array. A technique is given for calculation of number of photons arriving from different heights of the atmosphere from the detector response and, thus, to reconstruct the longitudinal development of individual showers. We present the results of direct observations of the cascade curve and give the depth of maximum shower development determined from the measurement of the Cherenkov emission from EAS. From the comparison between experimental values of $X_{\max }$ with simulation results based on the QGSJET-II-03 model for different primary particles, a preliminary conclusion was made on the cosmic rays mass composition in the energy range $10^{16}-10^{18} \mathrm{eV}$.

\section{${ }^{1}$ Speaker}




\section{Introduction and Experimental array}

The particle of cosmic rays entering the atmosphere from outer space reacts to the nuclei of air atoms and forms an avalanche process of secondary particles [1]. This phenomenon is called the extensive air shower (EAS). It is registered since the early 1970s. at the Yakutsk complex array [2]. The width of shower at the surface of the Earth can reach hundreds and thousands of meters, so the Yakutsk array consists of observation stations on the territory of $12 \mathrm{~km}^{2}$, which accommodate 2 scintillation counters, each of $2 \mathrm{~m}^{2}$, and a Cherenkov radiation receiver based on the photomultiplier (PMT-49). At present, 49 stations participating in the selection of events are located in a circle with a radius of $2 \mathrm{~km}$, so that a grid of regular triangles is formed. At 19 stations in a circle of $1 \mathrm{~km}$ radius, besides the scintillation counters, the Cherenkov EAS radiation receivers i.e. the Cherenkov integral detectors were installed. Inside this circle three muon detectors of $20 \mathrm{~m}^{2}$ each are located. In the center of array there is a large muon detector of $200 \mathrm{~m}^{2}$. As a part of the Yakutsk array, six antennae were installed to resume the recording of the radio EAS emission which are located at a distance of $300 \mathrm{~m}, 350 \mathrm{~m}$ and $500 \mathrm{~m}$ from the center of array [3]. At the Yakutsk EAS array a wide field-of-view Cherenkov telescope [4] develops which is operated by coincidence with the surface detectors of the extensive air shower array. Also, at a distance of $0.5 \mathrm{~km}$ from the center three tracking Cherenkov detectors (Obscura cameras) [5] were installed.

\section{Tracking Cherenkov detectors}

To investigate the longitudinal development of the shower at the Yakutsk EAS complex array the tracking Cherenkov detectors are used (see Fig.1). They consist of a camera, mosaic of photomultipliers, recording counter [6,7]. The Obscura 1 and Obscura 2 have narrow rectangular slits, Obscura2 has two narrow circular slits. The mosaic of Obscura 1 is a chain of photomultipliers located on the lower part of the plane of the camera along a line perpendicular to the slits. The mosaic of Obscura 2 and 3 has a cross-shaped form located also in the lower part of the plane of the camera.
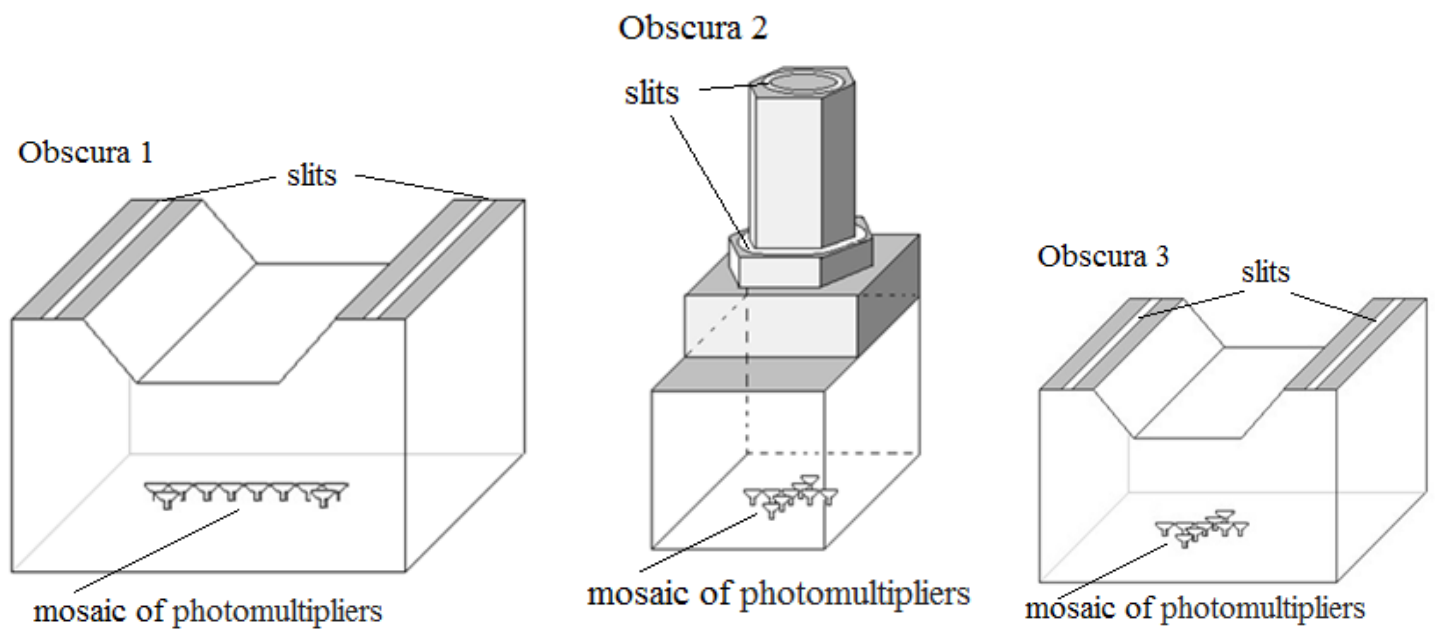

Figure 1: All 3 tracking Cerenkov detectors with different slits and mosaic of photomultipliers 
The peculiarity of the tracking Cherenkov detector operation is that the detector makes possible to receive information about the differential flow of the Cherenkov EAS light coming from different heights above sea level. A rather simple construction of the detector in the form of a camera with narrow slits has the narrow viewing angle along the vertical and widely possible angle of the horizontal coverage. The illuminated photocathode area of the photomultiplier and their number for this detector changes when the shower moves from the upper boundary of the site to the lower boundary (see Fig.2).

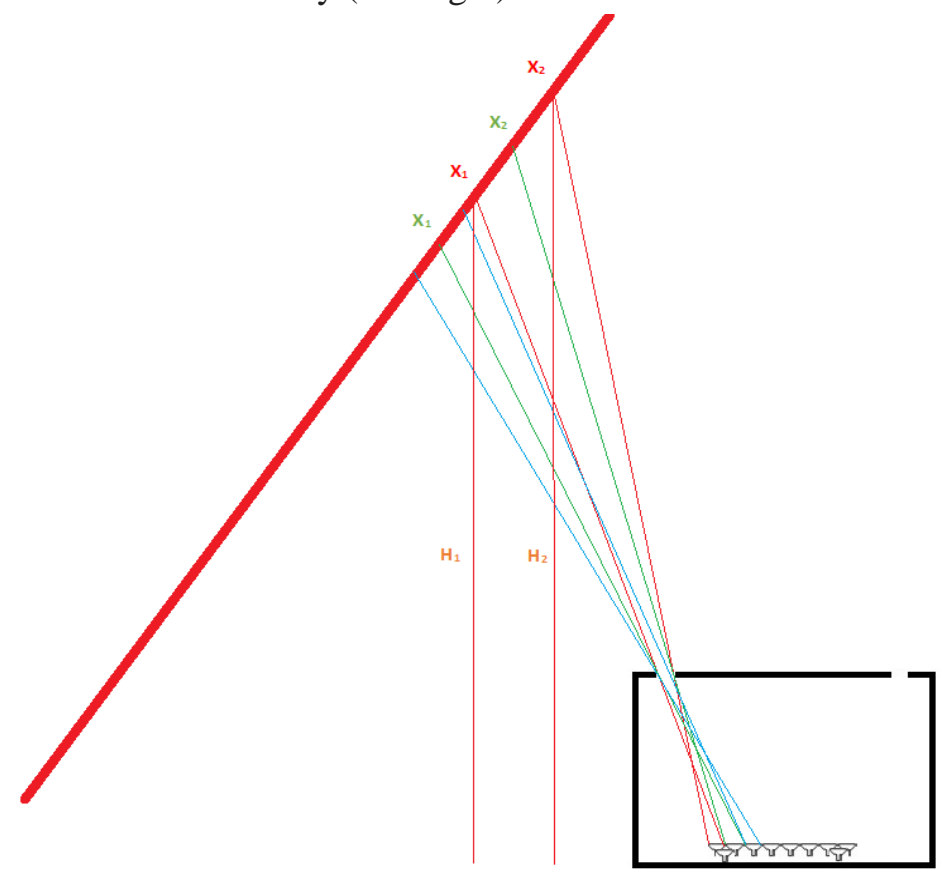

Figure 2: Image of the tracking Cherenkov detector and the boundaries surveyed by each photomultiplier of the mosaic $\Delta \mathrm{X}=\mathrm{X}_{2}-\mathrm{X}_{1}$

In each case, depending on the angles of arrival of the shower (azimuthal and zenithal angles) relative to this detector, the photomultiplier signals of the mosaic corresponding to different sections of the cascade curve of the EAS development will be received. Two slits in the case of the Obscura 1 and 3 detectors provide an overview of the section of the sky from different hemispheres along the azimuth angle. The construction of Obscura 2, due to two annular circular slits, covers a wider range of zenith angles and, because of the cross-shaped arrangement of the photomosaic, provides more or less the uniform efficiency of azimuth observations. 


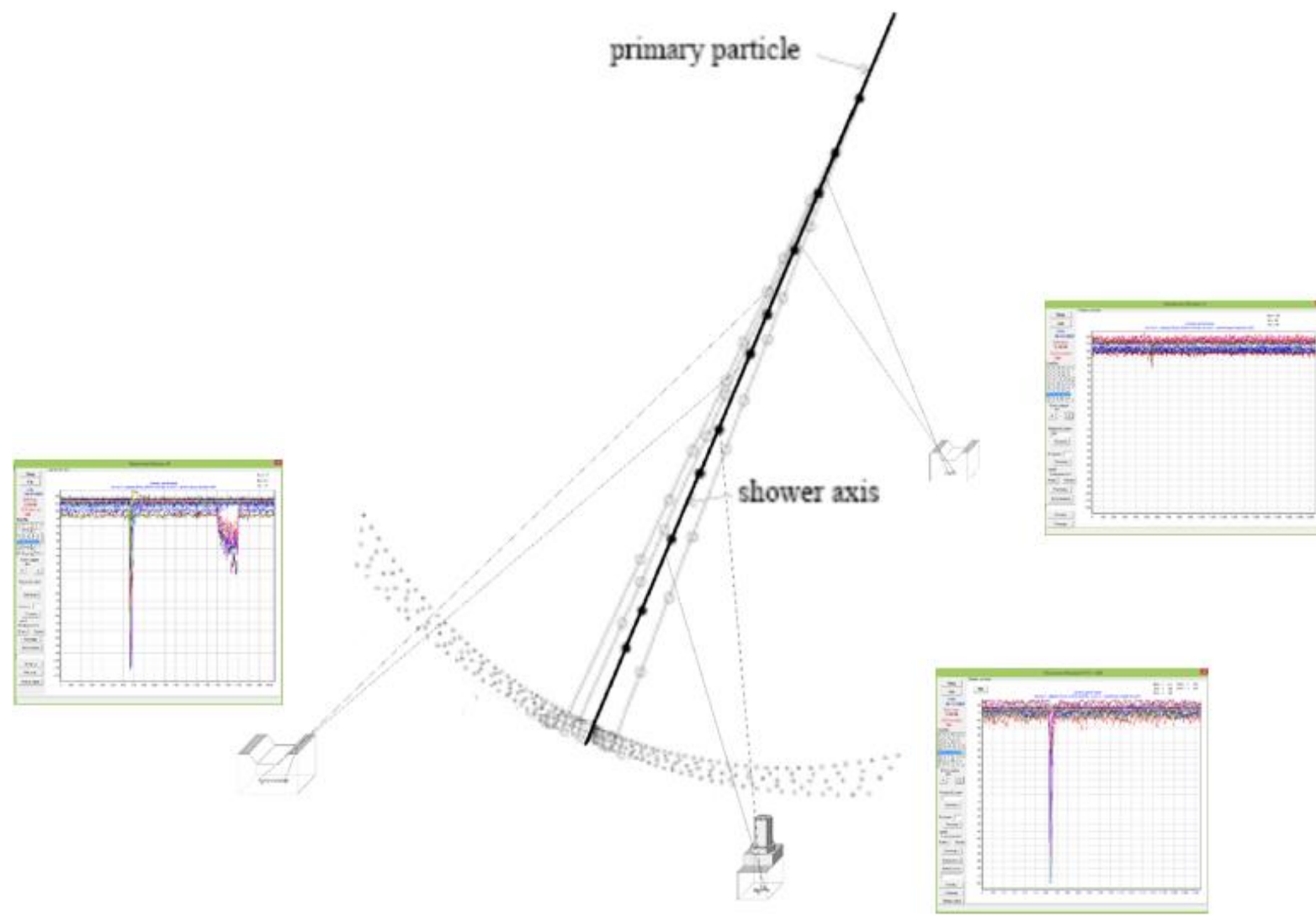

Figure 3: Example of recording a shower event with all three detectors

Figure 3 shows the simultaneous recording of a shower event with all three tracking detectors. In this event, in Obscura 1, in Obscura 2, in Obscure 3 eight, nine, six photomultipliers came into action, respectively. But in reality the statistics of such shower events is very small.

\section{Metodology for reconstruction of the cascade curve}

Using a methodology described in details in $[8,9,10]$, showers with energies above $10^{16} \mathrm{eV}$ were selected to reconstruct the cascade curves. In the showers a common charged component was recorded with the scintillation counters and the Cherenkov EAS light - with the integral and differential detectors. The main characteristics of the EAS are following: the shower arrival angles $\theta, \varphi$, the shower axis, the shower energy, the total number of charged particles at the observation level are determined by the indications of the main observation stations. The determination error of the axis coordinates at the Yakutsk EAS array is $15-35 \mathrm{~m}$, and the zenith angle of the direction of arrival is determined with the accuracy of about $3-5^{\circ}$. Unfortunately, such uncertainty in the localization of the shower axis leads to additional errors in the analysis of tracking detector data.

According to the program for processing of the recording data, the $H$-heights detected by each photomultiplier of the mosaic are determined by the tracking Cherenkov detector. Next, the total number of charged particles is calculated at the observation level by the data of large array scintillation detectors (see Fig.4). 


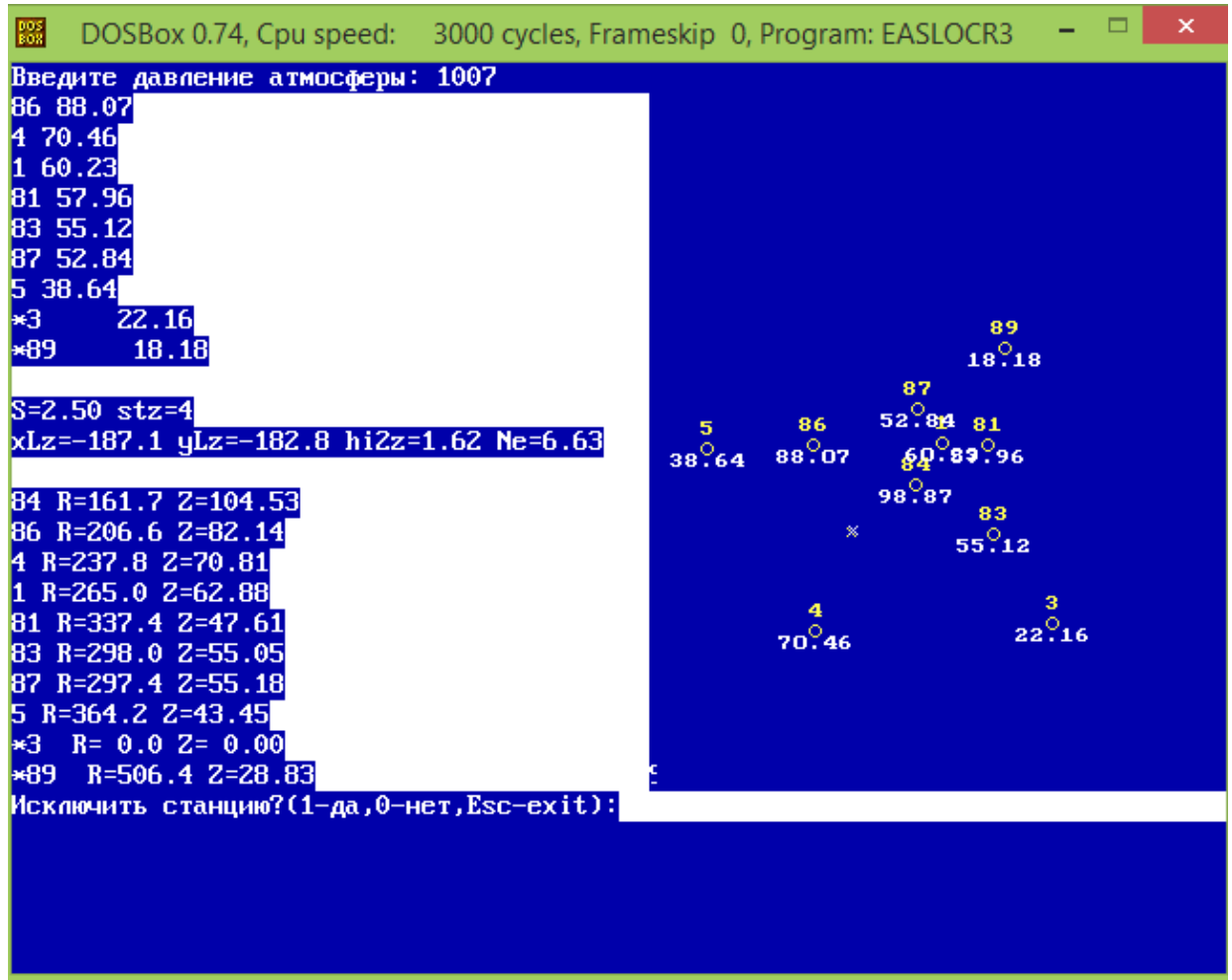

Figure 4: Determination of the number of particles $N_{s}$ at the observation level by the data of scintillation detectors

Furthermore, this EAS characteristic is used to construct the tail of cascade curve behind the depth of maximum shower EAS development and reconstruction of the cascade curve in the wide range in depth of the atmosphere. In minimizing of the experimental data, the formula (1) is used as the first approximation, in determining the number of particles at the mean depth in the atmosphere by the indications (amplitudes) of photomultipliers.

$$
N_{i}=N_{\max }+\left[0.434 \cdot\left(6.5+0.021 \cdot\left(X_{\max }-600\right)\right)\right] \cdot\left(1-\left(X_{i} / X_{\max }\right)+\ln \left(X_{i} / X_{\max }\right)\right)
$$

Where $N_{s}$ is the number of particles at the observation level, $X_{s}=1020 \cdot \sec \theta$ is the depth at the observation level depending on the zenith angle $\theta, \mathrm{N}_{\max }$ is the number of particles in the maximum of shower which is determined from the formula:

$$
N_{\max }=N_{s}-\left[0.434 \cdot\left(6.5+0.021 \cdot\left(X_{\max }-600\right)\right)\right] \cdot\left(1-\left(X_{s} / X_{\max }\right)+\ln \left(X_{s} / X_{\max }\right)\right)
$$

The iteration of the minimization process is stopped when the minimum condition is reached, that is, when a good agreement of the experimental data and the expected function (1) is reached. Thus, $X_{\max }$ and other parameters of the cascade curve are determined.

\section{Observation results and Conclusions}

All shower events have been selected in the tracking detector with the simultaneous operation of more than 8 photomultipliers of the mosaic for the 2006-2011 season with a master signal from a large array. By the method described above, the depth of the maximum and the number of particles of each shower event have been calculated. Further, these shower events have been separated in energy into three groups and have been marked by black dots in Figure 5. In this Figure, 
the obtained dependence $X_{\max }\left(E_{o}\right)$ is shown in comparison with calculations for the model QGSJETII-03 for the primary proton (red line) and iron core (blue line). In the energy range of $\sim 10^{17} \mathrm{eV}$, the black dots are grouped closer to the curve from the iron core, and above $7 \cdot 10^{17} \mathrm{eV}-$ it tends to approach the proton calculation.

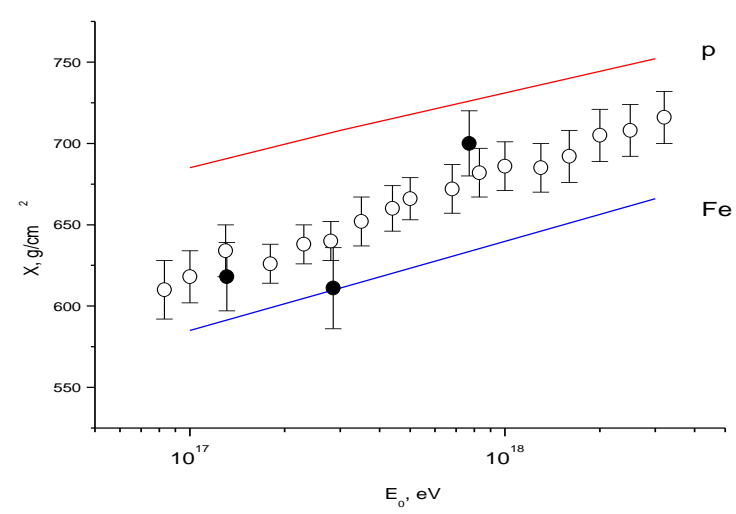

Figure 5: Dependence of $X_{\max }\left(E_{0}\right)$ is given in comparison with calculations for the model QGSJETII-03

White dots have been obtained according to the form of spatial distribution of the Cherenkov light at the Yakutsk EAS array. Consequently, within the framework of the chosen models, it can be assumed that the composition of cosmic rays in the energy interval $\sim 10^{17} \mathrm{eV}$ is heavier than at $\sim 10^{18} \mathrm{eV}$.

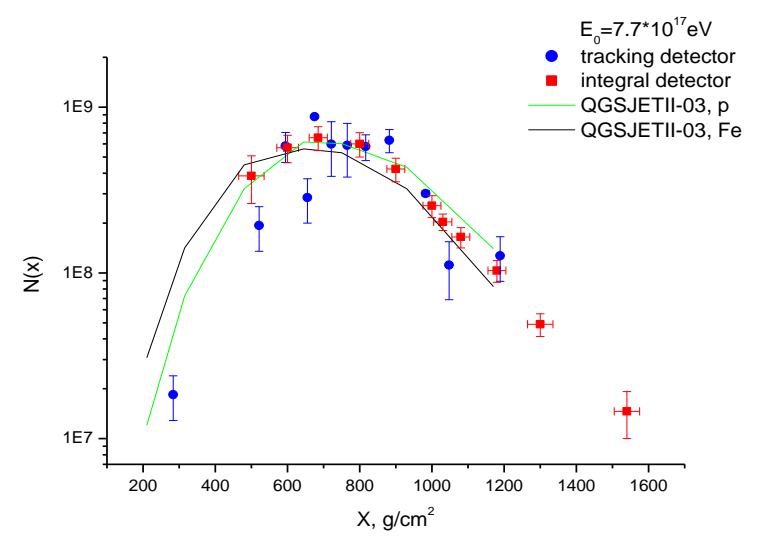

Figure 6: Dependence of the number of particles $N(x)$ on the depth of the $X$ of EAS development

The maximum development of EAS, found by direct measurements of the cascade curve for showers with $\left\langle E_{0}\right\rangle=7.7 * 10^{17} \mathrm{eV}$, is at a depth $\left\langle X_{\max }\right\rangle=656 \pm 62 \mathrm{~g} / \mathrm{cm}^{2}$ (see Fig.6). The calculations for the QGSJETII-03 model are also given for the case if a cascade of particles is generated by the primary proton and primary iron core. It is seen from Figure 5 and Figure 6 that the data of $X_{\max }$ lie between the calculated curves for the proton and iron core and rather correspond to the mixed composition of cosmic rays. And the fraction of iron nuclei can consist of 20 to $40 \%$. In order to make a more unambiguous conclusion, the extended set of statistics of showers is necessary which means that it is necessary to conduct further observations of the EAS using a tracking detector. 
The described construction of tracking detector based on the Obscura camera is more simple for making and operation, nevertheless, a high accuracy of measurement of all parameters is required to minimize the errors in determining the radiation flux density. For this aim, at present, the Yakutsk EAS array is modernizing for more accurate determination of localization of the shower axis that will allow to improve the analysis results for the calculation of measurements with the tracking detectors.

\section{Acknowledgements}

The work was carried out with the financial support of the grant "Scientific and Educational Fund for Support of Young Scientists of the Republic of Sakha (Yakutia)" №20170220235.

The author expresses a deep gratitude to the scientific researcher of IKFIA SB RAS Grigoriev Viktor Mikhailovich for his significant contribution to the formation of new method for investigation of the longitudinal development of extensive air showers.

\section{References}

[1] B. Rossi, Cosmic rays, Moscow 1966.

[2] Responsible editor, I.Ye. Sleptsov, Characteristics of extensive air showers of cosmic rays of ultrahigh energies, Yakutsk branch SB RAS Editions, Yakutsk 1976.

[3] S.P. Knurenko, Z.E. Petrov, I.S. Petrov, Radio emission of air showers with extremely high energy measured by the Yakutsk Radio Array, Nuclear Instruments and Method in Physics Research A. 866 (2017) 230-241

[4] A.A. Ivanov, S.P. Knurenko, A.D. Krasilnikov, Z.E. Petrov, M.I. Pravdin, I.Ye. Sleptsov, L.V. Timpfeev, Wide field-of-view Cherenkov telescope for the detection of cosmic rays in coincidence with the Yakutsk extensive air shower array, Nuclear Insctruments and Methods in Physics Research A 772 (2015) 34-42.

[5] G.K. Garipov, V.M.Grigoryev, N.N.Efremov et al. The Cherenkov Track Detector Consisting of the Yakutsk Complex EAS Array, 2001, ICRC, 885-887.

[6] Y.A. Egorov, S.P.Knurenko, A.V.Saburov, Origin of impulses delayed by $\tau \geq 5$ Ms in scintillation detectors for $E_{0} \geq 10^{18} \mathrm{eV}$ showers, 2013, Irkutsk, BSFP, 63-65.

[7] S.P. Knurenko, V. A.Kolosov, Z.E.Petrov et al.,2001, ICRC, 177.

[8] VM Grigoriev, A new method for investigating the longitudinal development of wide air showers // Science and Technology in Yakutia, Yakutsk, 2007, No. 2 (13), 28-33.

[9] V.P. Prokhorova, S.P. Knurenko, I.Ye. Sleptsov, A.V. Saburov, Longitudinal development of wide atmospheric showers in the energy region above $10^{15} \mathrm{eV}$ from observations of Cerenkov radiation by a camera obscura at the Yakutsk complex, 2009, BSFP, 319-321. 